\title{
Photophysics of Poly(p-Phenylene Vinylene) Films
}

\author{
M. Anni ${ }^{1}$, G. Gigli ${ }^{1}$, R. Cingolani ${ }^{1}$, Y. Galvão Gobato ${ }^{2}$, A. Vercik ${ }^{2}$, A. Marletta $^{3}$, \\ F.G. E. Guimarães ${ }^{4}$, and R. M. Faria ${ }^{4}$ \\ ${ }^{1}$ National Nanotechnology Laboratory, Dip. Ingegneria dell'Innovazione \\ Università degli Studi di Lecce, Via per Arnesano 73100 Lecce, Italy \\ ${ }^{2}$ DF-Universidade Federal de São Carlos, C.P. 676, 13565-905, São Carlos, SP, Brazil \\ ${ }^{3}$ FaFis-Universidade Federal de Uberlândia, C.P. 593, 38400-902, Uberlândia, MG, Brazil \\ ${ }^{4}$ IFSC-Universidade de São Paulo, C.P. 369, 13660-970, São Carlos, SP, Brazil
}

Received on 31 March, 2003

\begin{abstract}
We report on time resolved photoluminescence (PLRT) measurements in poly ( $p$-phenylene vinylene) (PPV) films irradiated by laser in the presence of air. We observe a PL intensity enhancement and a biexponential decay dynamics of PL signal for all irradiated films. These results can be understood in terms of a chain shortening process due to carbonyl incorporation and formation of an energy profile that extends and migrates into the film and enables efficient spectral diffusion of excited carriers to a non-degraded PPV segments by Förster energy transfer.
\end{abstract}

\section{Introduction}

Organic semiconductors such as poly(p-phenylene vinylene) (PPV) are of great interest due to their potential device applications such as light-emitting devices and photocells [14]. Many of the photophysical processes underlying the photodegradation of luminescent polymer devices have been satisfactorily explained [1-4]. Models of photoluminescence (PL) decay dynamics $[1,5,6]$ and photoluminescence excitation spectroscopy (PLE) [2,7-9] reveal details of carrier dynamics, quenching mechanisms and oxidation processes. The quenching process was accounted by attributing an additional non radiative decay channel to excitons [1-7]. This effect has been ascribed to the creation of carbonyl groups. However, PL intensity enhancement induced by a proper laser irradiation has been recently demonstrated in a PPV cast film [10]. This effect can be understood in terms of the chain shortening process due to carbonyl incorporation and formation of an energy profile that extends and migrates into the film and enables efficient spectral diffusion of excited carriers to a non-degraded PPV layer.

In this paper, we have studied time resolved photoluminescence in the picosecond time scale in poly(p-phenylene vinylene) (PPV) cast films upon laser irradiation in air with PL intensity enhancement. Several films of different thickness and different exposure times have been compared to fresh not irradiated samples. All the irradiated films show a biexponential decay dynamics with a fast non radiative process of about 50 ps and a longer decay time of about 250 ps. The PL efficiency is higher when the slow relaxation process is more important, thus indicating that the quick relaxation is non radiative. The radiative recombination importance increases in some irradiated samples, despite the increased defect density, indicating that a transfer process to a pure region is occurring.

\section{Experiment}

The poly(xylylidene tetrahydrothiophenium chloride) precursor was synthesized following a precursor route presented in Ref [11]. The precursor films were prepared by casting on hydrophobic substrates and were dried under vacuum for 24 hours. PPV-cast films were obtained by a thermal conversion at $230{ }^{\circ} \mathrm{C}$ under vacuum for 2 hours. The samples thicknesses characteristics used in this work are $0.68 \mu \mathrm{m}$ (samples A1,A2,A3), 0.98 $\mu \mathrm{m}$ (samples B1,B2,B3) and $1.51 \mu \mathrm{m}$ (sample $\mathrm{C} 1, \mathrm{C} 2, \mathrm{C} 3$ ). PPV-cast films were photoirradiated using a $458 \mathrm{~nm}$ Ar ion laser at several intensities under atmosphere conditions and room temperature. The PL spectra were recorded by a Spex 500M single spectrometer. A Coherent Ar ion laser was used as excitation source and the PL signal was detected by a photo counting system connected to a thermoelectrically cooled R5108 Hamamatsu photomultiplier. In the time-resolved photoluminescence experiments, the samples were excited by the second harmonic $(390 \mathrm{~nm})$ of a mode locked Ti-sapphire laser (Spectra Physics Tsunami) delivering 2 ps pulses at $780 \mathrm{~nm}$. The measurements were performed under vacuum. The PL signal was dispersed by a $0.24 \mathrm{~m}$ single monochromator coupled with a streak camera equipped with a twodimensional CCD. The time resolution was about $10 \mathrm{ps}$.

We have used a mask with $3.2 \mathrm{~mm}^{2}$ hole center area for all samples. We have also used a spot laser area with about 
$3.2 \mathrm{~mm}^{2}$. The PL measurements were performed to control the laser irradiation process for different films in air. The effects of the laser irradiation on time resolved photoluminescence have been studied in samples of different thickness and for different irradiation condition. The samples A1 $(0.68 \mu \mathrm{m}), \mathrm{B} 1(0.98 \mu \mathrm{m}), \mathrm{C} 1(1.51 \mu \mathrm{m})$ are non-irradiated samples. Samples A2 and A3 were irradiated during $35 \mathrm{~min}$ with laser intensities of $340 \mathrm{~mW} / \mathrm{cm}^{2}$ and $510 \mathrm{~mW} / \mathrm{cm}^{2}$, respectively. Samples B2, B3 were irradiated with laser intensity of $340 \mathrm{~mW} / \mathrm{cm}^{2}$ during $37 \mathrm{~min}$ and $8 \mathrm{~min}$, respectively. Samples C2 and C3 were also irradiated for laser intensity with $340 \mathrm{~mW} / \mathrm{cm}^{2}$ during $3 \mathrm{~min}$ and 16 min respectively. To verify the effect of the ambient light, since all the samples used in this work were exposed to this condition, the PLRT measurements were carried out in the irradiated and nonirradiated sample centers and in a region protected by a mask (unmasked region).

\section{Results and discussion}

Figure 1 shows typical infrared spectra for the PPV film before and after laser exposure. The insert displays the increase of the integrated PL intensity during the period of excitation. The increase of the infrared peak related to carbonyl group $\mathrm{C}=\mathrm{O}$ stretch at $1690 \mathrm{~cm}^{-1}$ suggests that a photo-oxidative reaction with carbonyl formation occurs during the PL enhancement process.

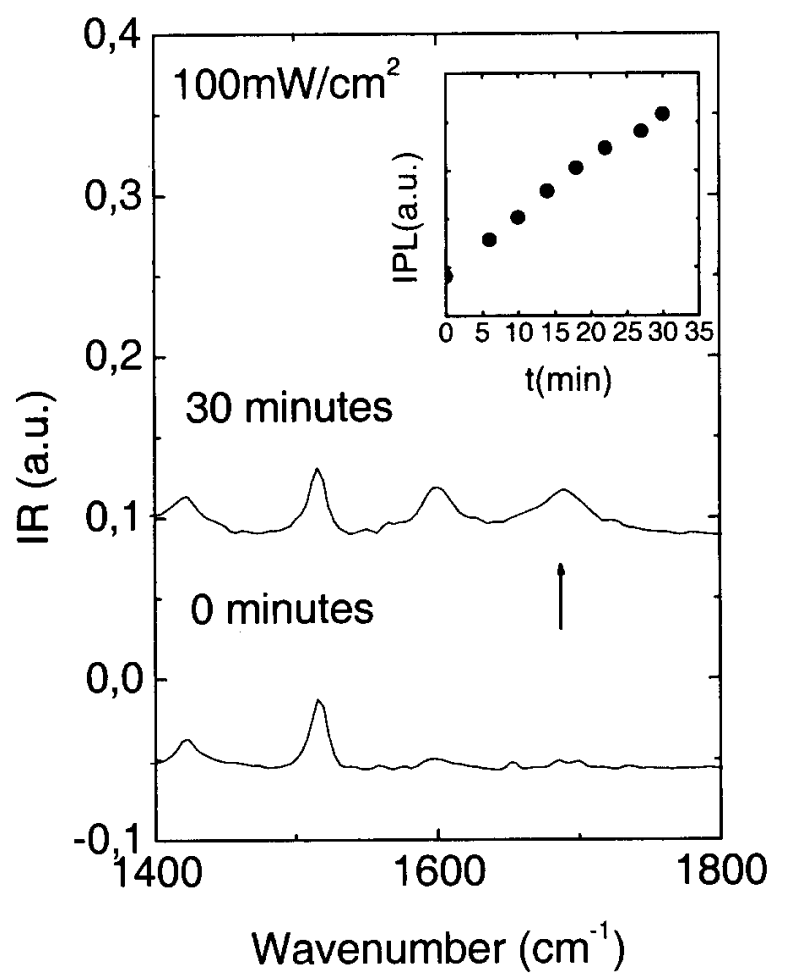

Figure 1. Typical infrared spectrum before and after photo irradiation during 30 minutes at $100 \mathrm{~mW} / \mathrm{cm}^{2}$. The insert shows the time dependence of PL integrated intensity.

The PL enhancement process depends strongly on the experimental constraints used, i.e., the ratio $d / \lambda_{o}$ between sample thickness $(d)$ and light penetration depth $\left(\lambda_{o}\right)$, which imposes a geometric limit for the defect formation and the carrier recombination [10]. The PL enhancement observed for film thickness $d \geq \lambda_{o}$ could be explained as follows. As the oxidation is not oxygen limited, the film is not uniformly oxidized. A carbonyl concentration profile is formed [7] following the laser intensity decay in accordance with the Beer-Lambert law. As a consequence of chain shortening process, an inhomogeneous profile of PPV segments of different sizes is continuously generated from the interface air/polymer into the film. The electronic structure of smaller segments is modified through the $\pi$-electron spatial localization, increasing the energy gap between the $\pi-\pi^{*}$ states [12]. Thus, the exciton energy differs from site to site along the film and an energy profile following the excitation intensity decay is built during irradiation. This energy profile and the strong overlap of vibrational states enables the carriers to relax energy efficiently through Förster transfer mechanisms $[1,9,13]$ towards sites of lower energy. This process favors the transfer of carriers to a non-degraded region in the bulk $[9,13]$; once in the non-degraded region, the carriers are confined in a thin layer due to the slow diffusion process [1] between long conjugated segments. The fact that shape and spectral position of PL emissions do not change over the entire temporal range we have studied is a strong evidence of carrier diffusion to a non-degraded layer. For the case of thin films $\left(d \leq \lambda_{o}\right)$, the degradation would extent across the whole film after the progressive migration of the degraded profile that reaches the interface film/substrate. The total PL emission begins to decrease as a consequence of the decrease of the effective absorption/emission thickness and of the recombination via carbonyl centers.

We have performed PLRT measurements under vacuum condition in the PPV films. In order to exclude the effects of defects due to the light exposure in air we used as a reference the measurements in the unmasked region of the nonirradiated sample. The PL spectra of the $0.68 \mu \mathrm{m}$ thick samples are reported in Fig. 2, while the time decay at the peak wavelength is reported in Fig. 3. A significant enhancement of PL intensity is observed for samples A2 and A3 irradiated during $35 \mathrm{~min}$ for laser intensity of $340 \mathrm{~mW} / \mathrm{cm}^{2}$ and $510 \mathrm{~mW} / \mathrm{cm}^{2}$, respectively. The PL decay show a biexponential decay with a longer time of $163 \pm 2$ ps and a faster relaxation with lifetime of $45 \pm 5$ ps for both samples. However in the irradiated samples the quenching on defects gives a smaller contribution to the relaxation, despite the increased defects density due to photo-oxidation during the laser irradiation. This feature is evidenced in the inset of Fig. 3, where the clear correlation between PL intensity and relative importance of the slow relaxation is shown. The decreased importance of the defect trapping despite the increased defect density indicates that some competitive relaxation process is transferring the excitation in a defect free region. As both samples have been exposed to laser light for a long time there will be a high defect density near the surface and a negative gradient deeper in the film, producing a negative gradient of conjugation length. This is the situation in which we can have energy transfer to the long chains far from the surface. The irradiation process gives also rise 
to some variation in the molecular geometry, evidenced by the slight line-shape variation in the low energy part of the PL spectrum, due to an oscillator strength redistribution between the different vibronic replicas of the 0-0 transition.

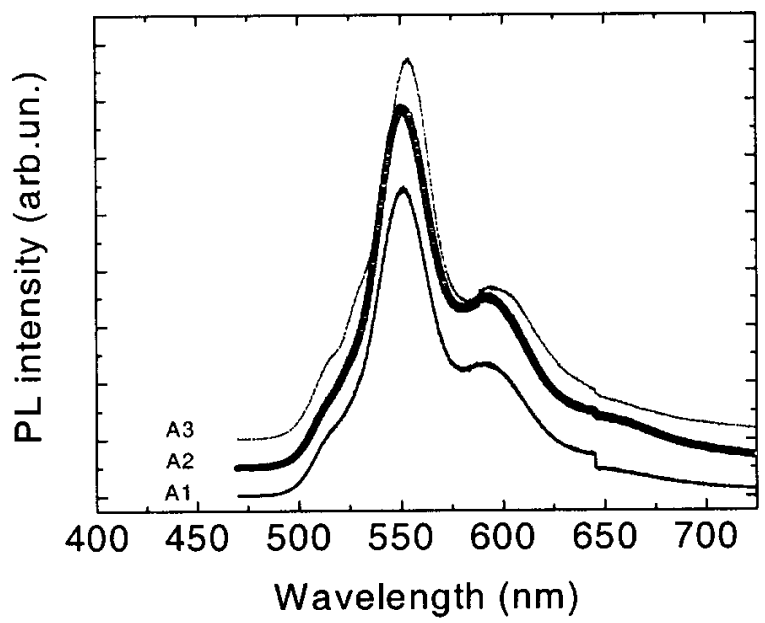

Figure 2. Time integrated PL spectra of samples A2, A3. The spectrum of sample A1 in the exposed to air region is reported as reference (continuous line).

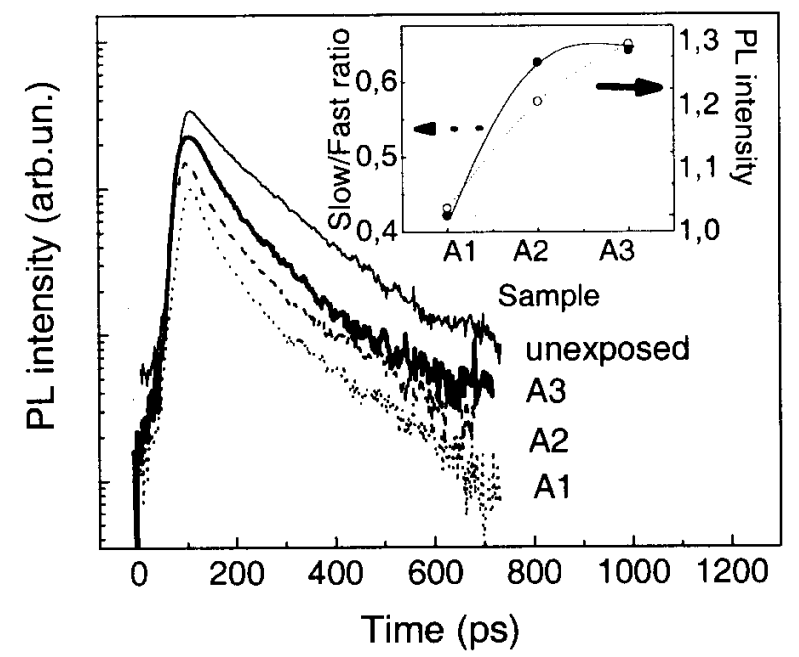

Figure 3. Transient PL dynamics at $550 \mathrm{~nm}$ of samples A1, A2, A3. The time resolved PL of sample A1 in a point not exposed to sun light is also reported (continuous line). The signal is multiplied for a normalization factor for clarity. Inset: Relative slow/fast relaxation weight (dotted line) and integrated PL intensity normalized to the reference sample (Alexposed to air). The lines are guide for the eyes.

We have similar results for samples B and C. Sample B3 irradiated for $8 \mathrm{~min}$ at laser intensity of $340 \mathrm{~mW} / \mathrm{cm}^{2}$ presents an increase of PL intensity of $29 \%$ with a biexponential PL decay with $60 \pm 3$ ps and $200 \pm 5$ ps decay times. The PL time constants are not affected by the irradiation but the importance of the slow relaxation increases again in the irradiated sample. The PL spectrum is slightly blue shifted by about $7 \mathrm{meV}$ indicating that the effective conjugation length in the emission region is increased. Sample B2 shows a strongly quenched PL(-59\%) together with a biexponential decay dynamic with unchanged time constants but with a lower relative slow/fast relaxation weight of 0.32 . This feature is again in agreement with your model, the defect density is high everywhere due to the longer irradiation and then the excitation mainly decays trough defect trapping. We have also a correlation between the emission intensity and the slow/fast relative weight.

Samples C2 and C3, irradiated for laser intensity of $340 \mathrm{~mW} / \mathrm{cm}^{2}$ during $3 \mathrm{~min}$ and $16 \mathrm{~min}$ respectively, show a PL spectrum with a more resolved vibronic progression. The PL intensity slightly decreases (about $9 \%$ ) in sample $\mathrm{C} 2$ indicating that something is changing in the nature of the emitting specie, which could be a morphological change, or a geometrical change of the molecule, which changes the exciton phonon coupling. We also observe a biexponential PL decay with $46 \pm 1$ ps and $235 \pm 7$ ps decay times. Sample C3 is in agreement with the trend observed in the series A and $\mathrm{B}$, the density of defects increases but a resonant transfer to the deep region of the film allows to obtain higher efficiency and higher contribution of the slow decay process. This sample presents a biexponential PL decay with $60 \pm 1$ ps and $210 \pm 7$ ps decay times. A similar correlation between PL intensity and relative importance of the slow relaxation is observed.

\section{Conclusions}

In conclusion, we have studied the photophysics of photo oxidized conjugated polymers for films with thickness that exceed the excitation penetration depth. The irradiated samples show a PL intensity increase despite the presence of photo-generated defects during the irradiation. We propose that these results can be explained in terms of the chain shortening process due to carbonyl incorporation and formation of an energy profile that extends and migrates into the film and enables efficient spectral diffusion of excited carriers to a non-degraded PPV layer. This model is confirmed by transient photoluminescence measurements showing in all the irradiated samples a biexponential decay with a fast decay time of about $50 \mathrm{ps}$ attributed to diffusion on defects and a slower decay time attributed to radiative recombination. In all the irradiated samples we observe that the PL enhancement is accompanied by a decrease of the importance of defects quenching. The decreased importance of the defect trapping despite the increased defect density indicates that some competitive relaxation process is transferring the excitation in a defect free region.

\section{Acknowledgements}

The authors acknowledge the financial support from FAPESP, FAPEMIG, CAPES and CNPq (Brazil).

\section{References}

[1] L. J. Rothberg, M. Yan, F. Papadimitrakopoulos, M. E. Galvin, E. W. Kwock, and T. M. Miller, Synth. Met. 80, 41 (1996). 
[2] A. T. H. Koch, N. T. Harrison, N. Haylett, R. Daik, W. J. Feast, and R. H. Friend, Synth. Met. 100, 113 (1999).

[3] S. M. Lipson, D. F. O’Brien, H. J. Byrne, A. P. Davey, and W. J. Blau, Thin Solid Films 370, 262 (2000).

[4] K. Tada, M. Onoda, J. Appl. Phys. 86, 3134 (1999).

[5] M. Yan, L. J. Rothberg, F. Papadimitrakopoulos, M. E. Galvin, T. M. Miller, Phys. Rev. Lett. 73, 744 (1994).

[6] R. Kersting, U. Lemmer, R. F. Mahart, K. Leo, H. Kurz, H. Bässler, and E. O. Göbel, Phys. Rev. Lett. 70, 3820 (1993).

[7] N. T. Harrison, G. R. Hayes, R. T. Philips, and R. H. Friend, Phys. Rev. Lett. 77, 1881 (1996).

[8] E. G. J. Staring, A. J. M. Berntsen, S. T. R. Romme, G. L. J. A. Rikken, and P Urbach, Phil. Trans. R. Soc. Lon. A 355, 695 (1997)
[9] M. C. J. M. Vissenberg, M. J. M. de Jong, Phys. Rev. B 56, 6681 (1997).

[10] Y. Galvão Gobato, A. Marletta, R.M. Faria, F.E.G. Guimarães, J.M. de Souza, and E.C. Pereira, Appl. Phys. Lett. 81, 942 (2002).

[11] A. Marletta, D. Gonçalves, O. N. Oliveira Jr., R. M. Faria, and F. E. G. Guimarães, Adv. Mater. 12, 69 (2000).

[12] W. Z. Wang, A. Saxena, and A. R. Bishop, Phys. Rev. B 50, 6068 (1994).

[13] M. C. J. M. Vissenberg, M. J. M. de Jong, Phys. Rev. Lett. 77, 4820 (1996). 